\title{
The Extent of The HI Halo in Five Isolated SB Galaxies
}

\author{
H. Kristen and S. Jörsäter \\ Stockholm Observatory S-13336 Saltsjöbaden, Sweden
}

\begin{abstract}
We investigate the extent of the HI halo in five isolated SB galaxies featuring strong bars. VLA ${ }^{1}$ observations of these objects are used to determine the diameter ratio between the $H I$ and the optical distribution. The mean diameter ratios $\mathrm{D}_{1.82} / \mathrm{D}(0)$ and $\mathrm{D}_{H I} / \mathrm{D}(0)$, for the sample presented here, are $1.1 \pm 0.1$ and $1.2 \pm 0.1$ respectively $(1 \sigma$ r.m.s.). For samples of various types, Bosma (1981) and Broeils (1992) find $\mathrm{D}_{1.82} / \mathrm{D}(0)=2.2 \pm 1.1$ and $\mathrm{D}_{H I} / \mathrm{D}(0)=1.8 \pm 0.4$ respectively. Our result thus could indicate compactness of the HI halo in galaxies with strong bars.
\end{abstract}

\section{Introduction}

The formation of a long-lived, bisymmetrical bar is a ubiquitous feature in $\mathrm{n}$ body simulations. Different possibilities for stabilizing against the bar instability have been suggested (e.g. Sellwood \& Wilkinson 1993 and references therein):

- Raising the velocity dispersion in the inner region.

- A sufficiently massive halo

- Interruption of a cycle consisting of amplification such as the 'swing amplifier' in combination with a feedback mechanism.

The purpose of this study is to investigate the possible connection between bars and HI halos by determining the diameter ratio between $\mathrm{HI}$ and the optical in a selected sample of barred galaxies. The extent of the HI is particularly interesting since it has in some cases been found to trace the dark matter (c.f. Broeils 1992). A correlation between the diameter ratio and type or luminosity has long been an issue of debate (e.g. Broeils 1992 and references therein).

\section{Selection and Observational Material}

A sample was selected by the following criteria:

\footnotetext{
${ }^{1}$ The VLA is operated by Associated Universities, Inc., under contract with the National Science Foundation
} 
- Barred systems

- Inclination $i \geq 20^{\circ}$, in order to select systems suitable for velocity mapping.

- $\mathrm{D}(0) \geq 2^{\prime}$, i.e. a diameter/beam ratio $\geq 6$ to provide spatial resolution.

- Numerical Hubble type (T) 1-6, corresponding to stages Sa to Scd, as defined in the Third Reference Catalogue of Bright Galaxies (de Vaucouleurs et al 1991, hereafter RC3)

These criteria were applied to the electronic version of the RC3, extracting 261 out of the over 23000 entries. The final criterion was:

- No visible interaction or known companions. This was assessed through visual inspection of $1^{\circ} \times 1^{\circ}$ fields extracted from the Digitized Sky Survey (Association of Universities for Research in Astronomy, Inc. 1993, 1994), in combination with the information on the NASA Extragalactic Database (NED). The purpose was to avoid non-selfconsistent systems.

Here we present results on five of these galaxies. Previous observations of NGC 1300 (England 1989, re-reductions by G. van Moorsel) and NGC 1365 (Jörsäter $\&$ van Moorsel 1995) are discussed as well as new observations of NGC 613, NGC 1350 and NGC 2263.

Table 1. Global Parameters

\begin{tabular}{llllll}
\hline & NGC 613 & NGC 1300 & NGC 1350 & NGC 1365 & NGC 2263 \\
\hline R.A. (2000) & $1^{\mathrm{h}} 34^{\mathrm{m}} 17^{\mathrm{s}} .5$ & $3^{\mathrm{h}} 19^{\mathrm{m}} 40^{\mathrm{s}} .8$ & $3^{\mathrm{h}} 31^{\mathrm{m}} 08^{\mathrm{s}} .4$ & $3^{\mathrm{h}} 33^{\mathrm{m}} 36^{\mathrm{s}} .6$ & $6^{\mathrm{h}} 38^{\mathrm{m}} 28^{\mathrm{s}} .3$ \\
Dec. (2000) & $-29^{\circ} 24^{\prime} 58^{\prime \prime}$ & $-19^{\circ} 24^{\prime} 41^{\prime \prime}$ & $-33^{\circ} 37^{\prime} 44^{\prime \prime}$ & $-36^{\circ} 08^{\prime} 17^{\prime \prime}$ & $-24^{\circ} 50^{\prime} 49^{\prime \prime}$ \\
Revised type (1) & SB(rs)bc & SB(rs)bc & $\left(\mathrm{R}^{\prime}\right) \mathrm{SB}(\mathrm{r}) \mathrm{ab}$ & $\mathrm{SB}(\mathrm{s}) \mathrm{b}$ & $\left(\mathrm{R}^{\prime}\right) \mathrm{SB}(\mathrm{r}) \mathrm{ab}$ \\
T (1) & 4.0 & 4.0 & 1.8 & 3.0 & 2.1 \\
$i\left(^{\circ}\right)$ & $41(1)$ & $35 \pm 4(2)$ & $58(1)$ & $55(3)$ & $54(1)$ \\
$P . A .\left(^{\circ}\right)$ & $120(1)$ & $266 \pm 2(2)$ & $0(1)$ & $220(4)$ & $143(1)$ \\
$\mathrm{V}_{21}$ & $1475 \pm 5(1)$ & $1568 \pm 6(1)$ & $1890 \pm 9(1)$ & $1662 \pm 4(1)$ & $2743 \pm 2(5)$ \\
\hline
\end{tabular}

(1) de Vaucouleurs et al. (1991)

(2) Kristen \& Lindblad (1995, in prep.)

(3) Lindblad (1978)

(4) Jörsäter \& van Moorsel (1995)

(5) Bottinelli et al. (1992)

Table 2. VLA Observations

\begin{tabular}{llll}
\hline & NGC 613 & NGC 1350 & NGC 2263 \\
\hline Date & $10 / 94$ & $10 / 94$ & $10 / 94$ \\
Configuration & CnB & CnB & CnB \\
Length (minutes) & 330 & 248 & 325 \\
Beam FWHM (") & $22.13 \times 14.33$ & $22.29 \times 13.79$ & $23.33 \times 14.19$ \\
\hline
\end{tabular}




\section{Results}

In order to facilitate comparison with the literature, we determine the diameter of the HI face-on flux density isophotes at two levels: $D_{1.82}$ (corresponding to $1.82 \times 10^{20}$ atoms $\mathrm{cm}^{-2}, 100 \mathrm{~K} \mathrm{~km} \mathrm{~s}^{-1}$ or $\left.1.44 \mathrm{M}_{\odot} \mathrm{pc}^{-2}\right)$, and $\mathrm{D}_{H I}\left(1 \mathrm{M}_{\odot} \mathrm{pc}^{-2}\right)$. The flux as a function of diameter is determined by averaging the total flux in inclination-corrected $10^{\prime \prime}$ thick concentric rings. The error estimate of the isophote diameter is an assessment of the diameter uncertainty associated with the patchiness of the HI distribution. $\mathrm{D}(0)$, as listed in RC3, is the diameter of the face-on corrected $25 \mathrm{~m}_{B} \operatorname{arcsec}^{-2}$ isophote along the major axis, compensated for extinction. The mean diameter ratios $\mathrm{D}_{1.82} / \mathrm{D}(0)$ and $\mathrm{D}_{H I} / \mathrm{D}(0)$ for the sample of five galaxies presented here, are $1.1 \pm 0.1$ and $1.2 \pm 0.1$ respectively (1 $\sigma$ r.m.s.). In comparison, Bosma. (1981) and Broeils (1992) find $\mathrm{D}_{1.82} / \mathrm{D}(0)=2.2 \pm 1.1$ and $\mathrm{D}_{H I} / \mathrm{D}(0)=1.8 \pm 0.4$ respectively, for samples of various types. Although the sample is small, this might point toward a connection between the HI halo (and contained mass) and development of bars.

Table 3. Results

\begin{tabular}{llllll}
\hline & $\begin{array}{l}\mathrm{D}_{1.82} \\
\text { arcmin }\end{array}$ & $\begin{array}{l}\mathrm{D}_{H I} \\
\text { arcmin }\end{array}$ & $\begin{array}{l}\text { Optical Diameter D }(0) \\
\text { arcmin }\end{array}$ & $\mathrm{D}_{1.82} / \mathrm{D}(0)$ & $\mathrm{D}_{H I} / \mathrm{D}(0)$ \\
\hline NGC 613 & $5.4 \pm 0.3$ & $5.8 \pm 0.3$ & $\mathbf{5 . 5 \pm 0 . 1 ( 1 )}$ & $\mathbf{1 . 0 \pm 0 . 1}$ & $1.1 \pm 0.1$ \\
NGC 1300 & $7.0 \pm 0.3$ & $7.5 \pm 0.3$ & $6.2 \pm 0.3(1)$ & $1.1 \pm 0.1$ & $1.2 \pm 0.1$ \\
NGC 1350 & $5.7 \pm 0.3$ & $6.0 \pm 0.3$ & $5.3 \pm 0.1(1)$ & $1.1 \pm 0.1$ & $1.1 \pm 0.1$ \\
NGC 1365 & $\mathbf{1 2 . 3 \pm 0 . 6}$ & $\mathbf{1 2 . 7 \pm 0 . 6}$ & $11.2 \pm 0.1(1)$ & $\mathbf{1 . 1} 0.1$ & $1.1 \pm 0.1$ \\
NGC 2263 & $4.2 \pm 0.2$ & $4.6 \pm 0.2$ & $3.2 \pm 0.1(1)$ & $1.3 \pm 0.1$ & $1.4 \pm 0.1$ \\
\hline
\end{tabular}

(1) de Vaucouleurs et al. (1991)

Acknowledgments. We thank Martin England for generously providing us with his HI data of NGC 1300 , and Gustaaf van Moorsel for performing the corresponding re-reductions.

\section{References}

Bosma, A. 1981, AJ, 86, 1825

Bottinelli, L., Durand, N., \& Fouqué , P. et al. 1992, A\&AS, 93, 173B

Broeils, A. 1992, PhD Thesis, University of Groningen

England, M. N. 1989, ApJ, 337, 191

Jörsäter, S. \& van Moorsel, G. 1995, AJ, 110, 2037

Lindblad, P. O. 1978, in Astronomical Papers dedicated to Bengt Strömgren, A. Reiz \& T. Andersen, Copenhagen University Observatory, 402

Sellwood, J. A. \& Wilkinson, A. 1993, Rep. Prog. Phys, 56, 173

de Vaucouleurs, G., de Vaucouleurs, A., Corwin, H. G. Jr., Buta, R. J., Paturel, G., \& Fouqué, P. 1991, Third Reference Catalogue of Bright Galaxies, New York: Springer 